تقطيع مرئيات التحس النائي إلى أنواع مختلفة من الأغطية الأرضية باستخدام طريقة حد العتبة (Otsu)

$$
\begin{aligned}
& \text { ضياء حازم سليمان البرهاوي } \\
& \text { مركز التحسس النائي } \\
& \text { جامعة الموصل } \\
& \text { (تاريخ الاستلام 2012/9/30 تاريخ القبول 2014/6/3 ) } \\
& \text { الملخص }
\end{aligned}
$$

تعتبر عملية تقطيع المرئيات من المراحل الأولية المهمة من مراحل إعداد قواعد البيانات لنظم المعلومات الجغرافية (GIS). حيث تم في هذا البحث استخدم طريقة حد العتبة (Otsu thresholding) لإنجاز عملية تقطيع المرئيات الفضائية (Image segmentation) نظرا لكونها طريقة سريعة وكفؤة وتقلل التباين ضمن الصنف الواحد. حيث نم تطبيق الدراسة لمعالجة أحد المرئيات التي تحتوي على نوعين رئيسيين

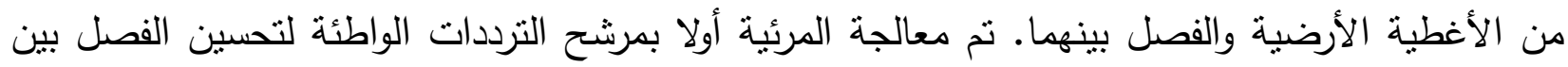
الأصناف الموجودة ضمن المرئية الواحدة، ثم انجاز عملية التقطيع بواسطة استخدام طريقة حد العتبة Otsu.

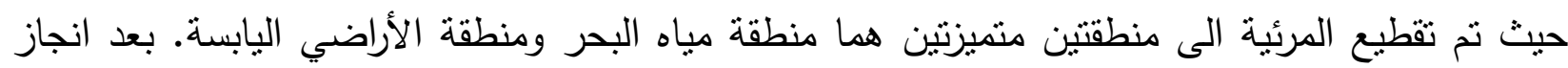

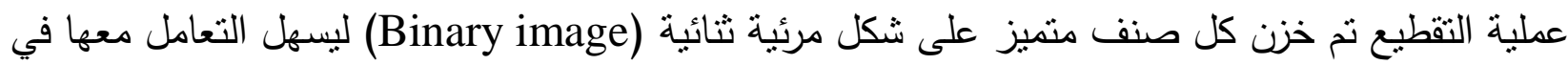
عمليات المعالجة الرقمية اللاحقة. الكلمات الدالة: تقيع المرئيات الفضائية، طريقة حد العتبة، المرئية الثنائية.

\title{
Segmenting of Remote Sensing Images into Different land Cover Types using Otsu Thresholding
}

\section{Dheyaa hazim sulaman AL-Barhawi \\ Remote Sensing Center \\ University of Mosul}

\begin{abstract}
Image segmentation is an important initial operation before building databases for GIS use. Otsu method is a fast and efficient thresholding approach because it is minimizing within class variance. In this study image segmentation has been done by using Otsu thresholding. First, an image contains two main cover types processed by Low Pass filter to enhance separation between classes.
\end{abstract}


Then, use thresholding to segment image into distinct regions (classes), water and land cover. Finally, each distinct region will store in binary image to uses in later image processing.

Keywords: Segmenting of remote sensing, thresholding approach, binary image.

\section{المقدمة}

تعتبر عملية تقطيع المرئيات من المراحل الاولية المهمة من مراحل اعداد قواعد بيانات نظم

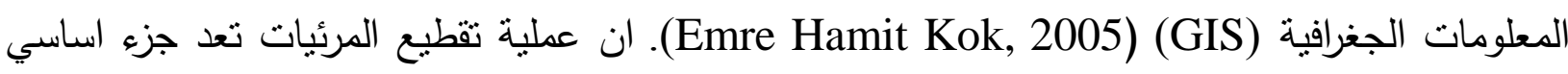
ومهم لبناء اي نظام الي لتمييز المرئيات الفضائية (Automatic image recognition system). وينظر الى عملية تقطيع المرئيات في تطبيقات الروية الالية (machine vision) على انها الجسر الذي لئي

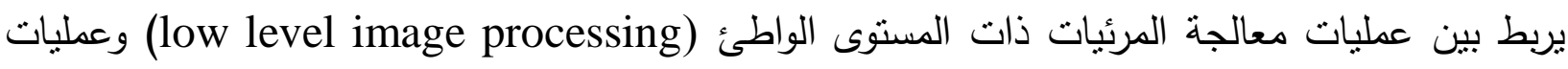
معالجة المرئيات ذات المستوى العالي (high level image processing). بينما ينظر الى عملية تقطيع الجئي المرئيات في تطبيقات التحس النائي على انها الاداة المساعدة التي تستخدم في عملية ايجاد وتقييم

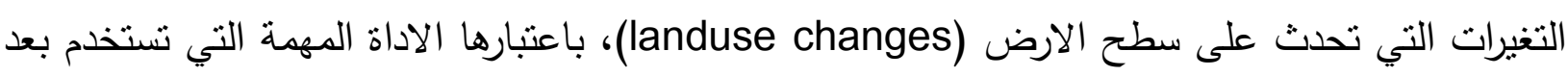
انجاز عملية تكوين الاصناف لتجزئة المرئية الى كيانات متميزة يمكن حساب مساحتها وقطرها ومحيطها .(Dey V. et al., 2010)

يمكن تعريف عملية تقطيع المرئيات او الصور الفضائية (Image Segmentation) بصورة عامة على انها المعالجة التي تؤدي الى تجزئة المرئية الى مناطق متجانسة (Homogenous Regions) بحيث لا يوجد منطقتين متجاورتين متجانستين وكما يلي:

$U S_{i}=F$ with $\quad S_{i} \cap S_{i}=\varnothing, i \neq j$

$i=1 . . n$

$j=1 . . n$

حيث F تمثل المرئية، اخر يمكن القول ان المرئية الاصلية Fمكن تكوينها من ناتج اتحاد (U) جميع المناطق المتميزة

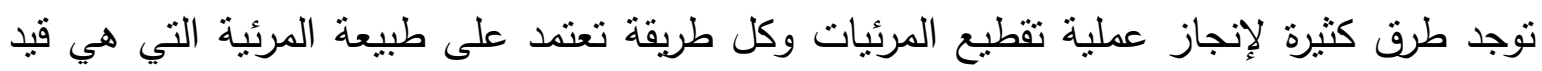

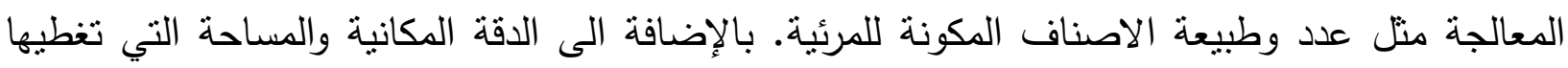

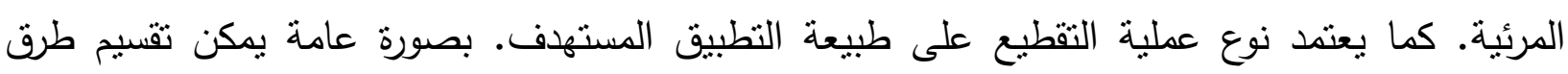

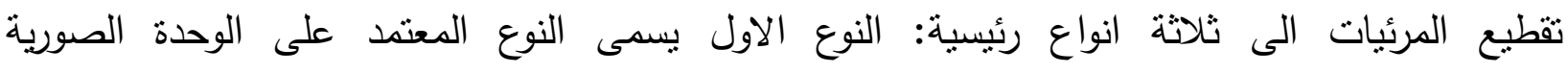
(Pixel-based methods) (Region-based methods) .(Edge-based methods) 
النوع الاول (Pixel-based methods) يعتبر ابسط انواع طرق تقطيع المرئيات من ناحية

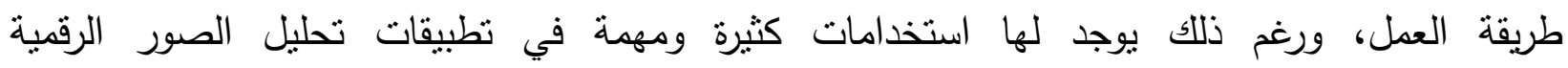
(digital image analysis)

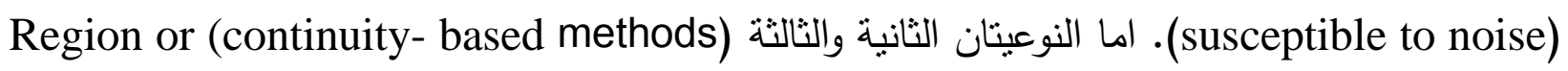
و تستخدمان مفهومين مختلفين لإنجاز عملية تقطيع المرئيات، حيث (Edge-based methods) تستخدم النوعية الثانية مفهوم التشابه Similarity بينما تستخدم النوعية الثالثة مفهوم الاختلاف (Dohn L. Semmlow, 2004) (Difference) تم في الدراسة الحالية الاعتماد على طريقة (Otsu) والتي هي من نوع (Pixel-based methods) في انجاز عملية تقطيع المرئية المستخدمة 1 - 1 Tريقة حد العتبة Thresholding لتقطيع المرئيات:

تعتبر طريقة حد العتبة باستخدام الوحدات الصورية من أهم الطرق وأكثرها شيوعا في تقطيع المرئيات التي تصنف ضمن الطرق المسماة (Pixel-based methods). حسب هذه الطريقة جميع الوحدات

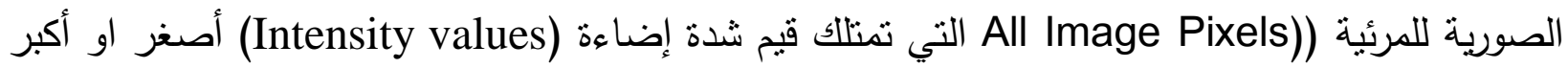
من قيمة حد العتبة تصنف على أنها تقع ضمن منطقة متجانسة واحدة (region homogenous). طريقة

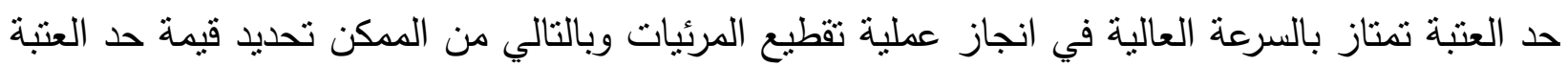

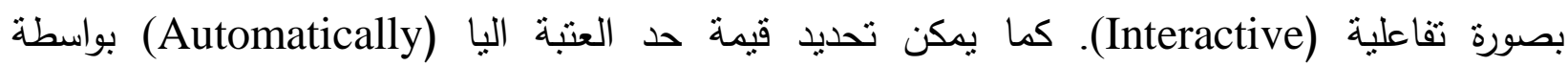
خوارزميات إيجاد حد العتبة. (Nikhil R. Pal. et al., 1993). تعتبر عملية تحديد قيمة حد العتبة من أول واهم وأصعب خطوات خوارزميات تقطيع المرئيات. حيث يستخدم المدرج التكراري للمرئية (Image Histogram) كأداة مساعدة لتحديد قيمة حد العتبة. ويمثل المدرج التكراري للمرئية التوزيع الإحصائي (statistical distribution) لقيم شدة الإضاءة لنقاط المرئية. ويستخدم المدرج التكراري أيضا لتقييم كفاءة خوارزمية نقطيع المرئية عندما نظهر قمم المدرج (Histogram peaks)

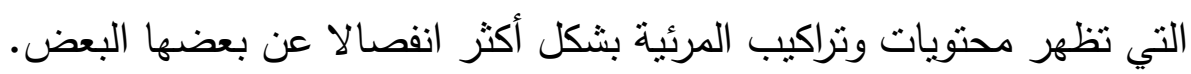

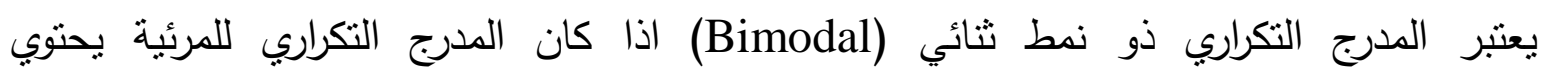
على قمتين (Peaks) أبي أن المرئية تحنوي على منطقتنين منميزتين ويعتبر المدرج التكراري ذو نمط دائي

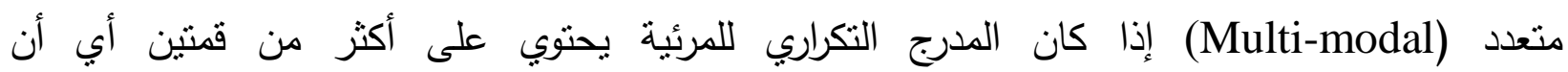
المرئية تحتوي على أكثر من منطقتين متميزتين. لتحديد قيمة حد العنبة يتم البحث عن أكثر الأماكن انخفاضا (Valleys) أو أكثر الأماكن ارتفاعا (Peaks) في المدرج التكراري للمرئية.

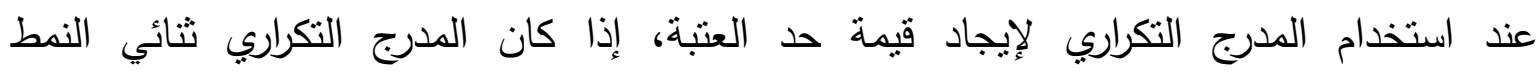

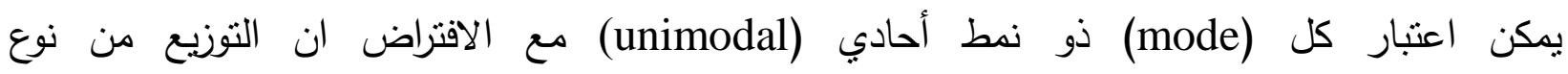
(Gaussian distribution) 


$$
\text { ضياء حازم سليمان البرهاوي }
$$

نقطة الثقاطع بين التوزيعين أفضل قيمة لحد العتبة. المشكلة الرئيسية لهذه الطريقة ان توزيع بيانات المرئية ليس دائما من نوع (Gaussian distribution). يوجد اسلوب اخر لإيجاد قيمة حد العتبة باستخدام المدرج التكراري. وفق هذا الاسلوب يتم ايجاد قيمة حد العثبة التي تقلل التباين (minimize variance) ضمن كل منطقة متميزة من المناطق التي تكون المرئية وقد استخدمت هذه الطريقة في هذا البحث كما سيتم توضيح تفاصيل عن هذه الطريقة في الفقرة

\section{2- 2 طtsu لاختيار قيمة حد العتبة:}

تعتبر طريقة Otsu من الطرق السهلة والكفؤة لتحديد قيمة حد العتبة بصورة الية (Automatic) وبالتالي لتقطيع وتجزئة المرئية الى مناطق متميزة ومتجانسة، بحيث ان كل منطقة متجانسة تمثل احد الاصناف (Classes) الموجودة في المرئية (Otsu N., 1979). ان طريقة (Otsu) لها نطبيقات واسعة في معالجة الصور الرقمية وخصوصا في مجال التحس النائي وفي المجالات الطبية (Medical Image Processing). من محاسن هذه الطريقة السرعة وسهولة

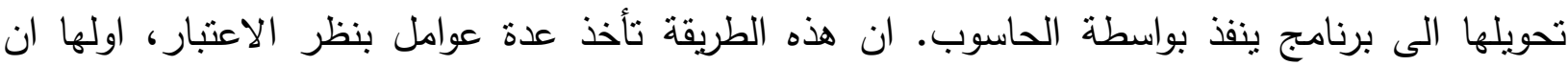

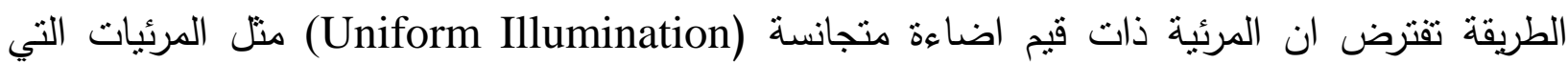
تحتوي على مساحات واسعة من الاراضي المزروعة او مساحات واسعة من الاراضي الصحراوية او

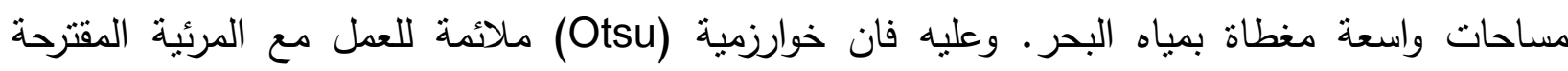

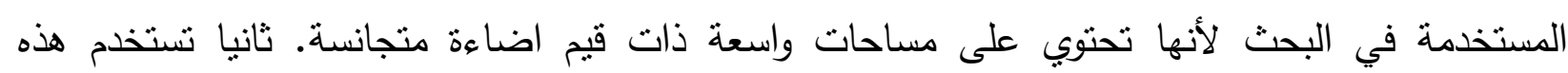

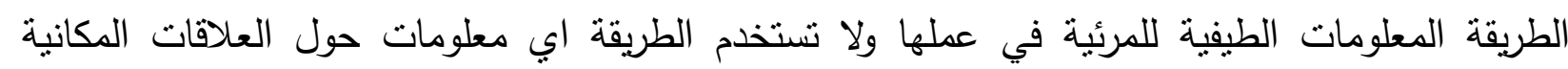
لمكونات المرئية في عملها. (Spatial relationships) يتم تحديد قيمة حد العتبة في هذه الطريقة بالاعتماد على شكل المدرج التكراري للمرئية حيث يفترضئ فئرض

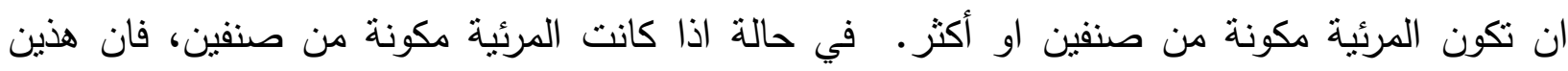

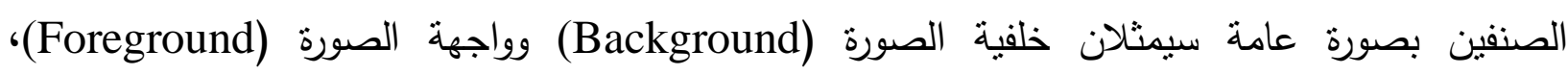
اما وفق مفاهيم التحسس النائي كل صنف سيمثل احد انواع الاغطية الارضية (Landcover types)،

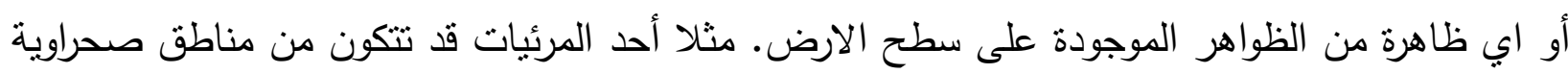

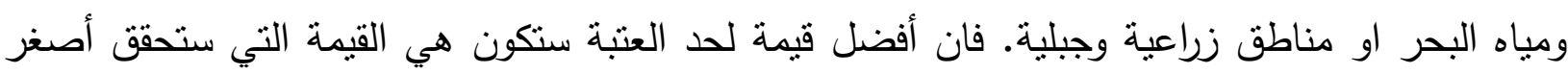
قيمة للتباين ضمن كل صنف (Otsu N, 1979) (minimizing within class variance). لتكن 1q و q2 تمثل المدرج التكراري لكل صنف من اصناف المرئية P وكما يلي :

$q_{1}(t)=\sum_{i=1}^{t} P(i)$ 
$q_{2}(t)=\sum_{i=t+1}^{I} P(i)$

(Otsu N, 1979)

ويتم تعريف التباين (Variance) لكل صنف في المرئية كما يلي :

$\sigma_{1}^{2}(t)=\sum_{i=1}^{I}\left[i-\mu_{1}(t)\right]^{2} \frac{P(i)}{q 1(t)}$ 4

$\sigma_{2}^{2}(t)=\sum_{i=t+1}^{I}\left[i-\mu_{2}(t)\right]^{2} \frac{P(i)}{q 2(t)}$

حيث 21 , م1 نمنل التباين.

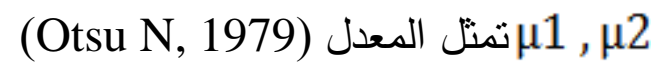

i تمثل قيمة شدة الاضاءة لكل نقطة من نقاط المرئية. يتم حساب معدل قيم النقاط

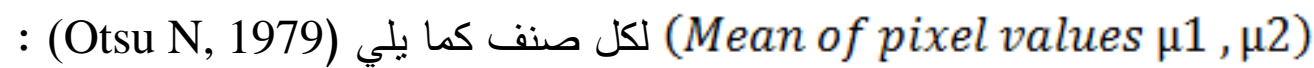

$\mu 1(t)=\sum_{i=1}^{t} \frac{i P(i)}{q 1(t)}$

$\mu 2(t)=\sum_{i=i+1}^{I} \frac{i P(i)}{q_{2}(t)}$

$a^{2}(t)=\sigma^{2}-\sigma^{2}(t)=q_{t}[1-$

$\left.q_{1}(t)\right]\left[\mu_{1}(t)-\mu_{2}(t)\right]^{2}$. $\ldots 8$

Minimizing within ) رياضيا يمكن تحويل مسألة ايجاد اقل تناين ضمن كل صنف (classvariance

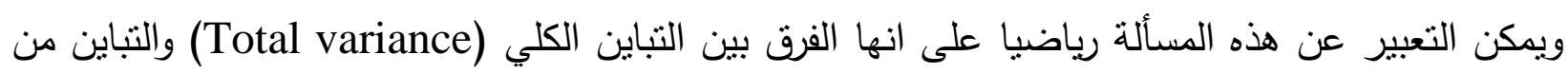

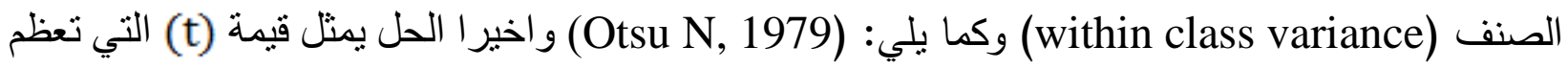
. (Otsu N, 1979) $\mathrm{a}^{2}(\boldsymbol{t})$ (Maximize) من المكن ان تحتوي المرئية على اكثر من منطقتين متميزتين لذا قام احد الباحثين بتطوير طريقة

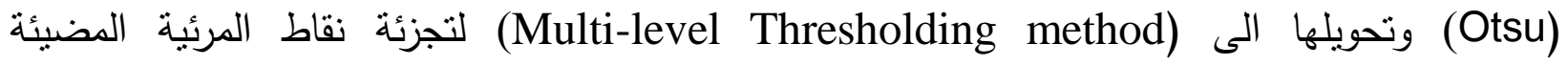
(Pixels) للمرئية يحتوي على ثلاث قمم (Peaks)، عند ذللك بمكن تقطيع المرئية باستخدام قيمنين لحد العتبة. هاتين

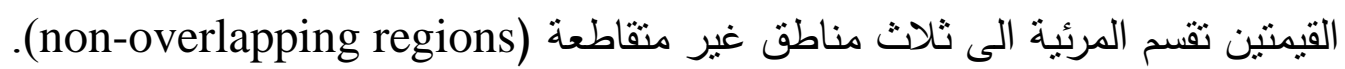

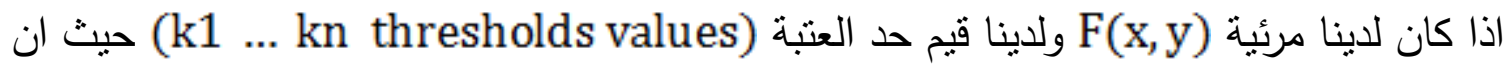
$\mathrm{k}_{1}>\mathrm{k}_{2}>\ldots>\mathrm{k}_{\mathrm{n}}$ 


$$
\text { ضياء حازم سليمان البرهاوي }
$$

بحيث يتم تحديد قيم حد العتبة k حسب طبيعة المرئية المستخدمة وحسب نوعية التطبيق. وبالتالي يتم

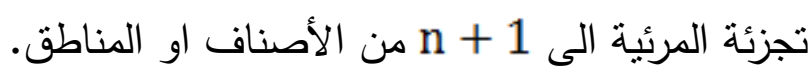

$$
\text { 3- - 3 منهج العمل: }
$$

المرئية المستخدمة في هذا البحث ملتقطة لمنطقة البحر الأحمر والتي تحتوي على نوعين من الأغطية الراضية كما في (الثكل 2)، المناطق التي تظهر باللون الاسود تمنل المياه وبقية المناطق تمنل اليابسة.

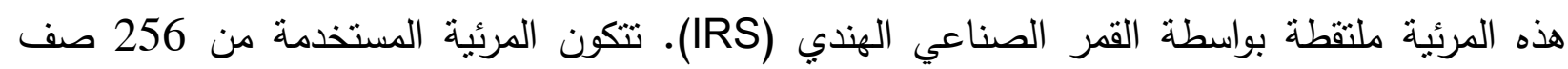

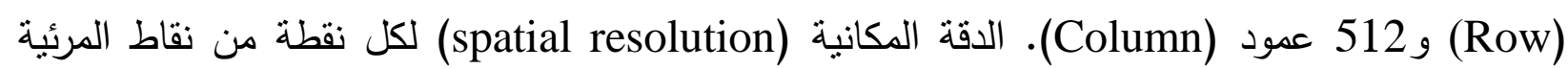

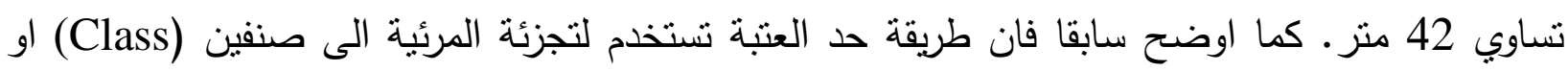
أكثر حسب مفاهيم التحس النائي. حيث تم في بداية العمل معالجة المرئية بمرشح كاوس لأمرار الترددات

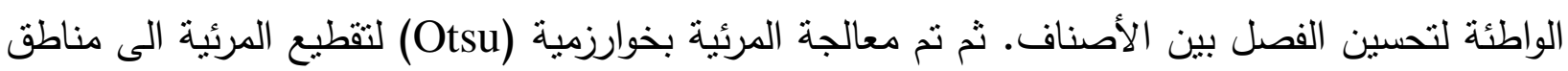
منميزة. أخيرا نم خزن كل منطقة منميزة ناتجة من عملية التقطيع في مرئية ثنائية (Binary Image) منفصلة. وكما في المخطط التالي (الثكل 1):

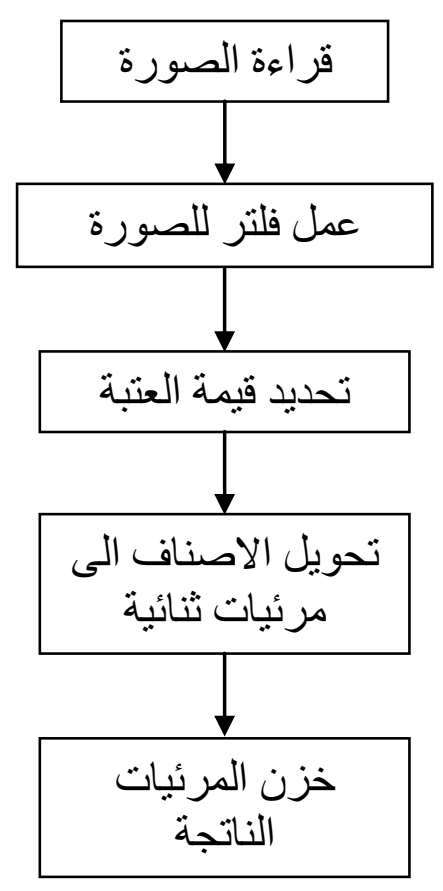

الثكل 1: خطوات وهيكلية العمل.

النتائج

ضمن خطوات البحث تم معالجة المرئية بواسطة مرشح كاوس لإمرار الترددات الواطئة

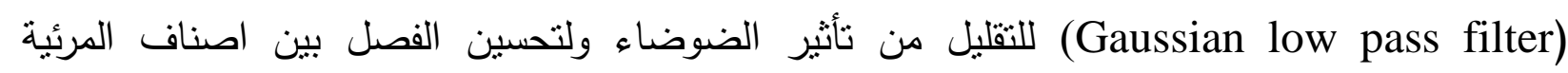
باستخدام طريقة (Otsu)، لاحظ (الثكل 2) حيث ان المدرج التكراري للمرئية قبل معالجتها بمرشح 
كاوس يحتوي على توزيع ثنائي النمط (bi-modal) والمدرج التكراري للمرئية بعد معالجتها بمرشح كاوس

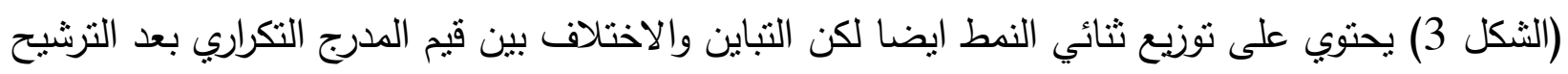
اصبح اكبر وبالتالي فان الفصل بين الاصناف يمكن ان يكون ادق وافئ لئل
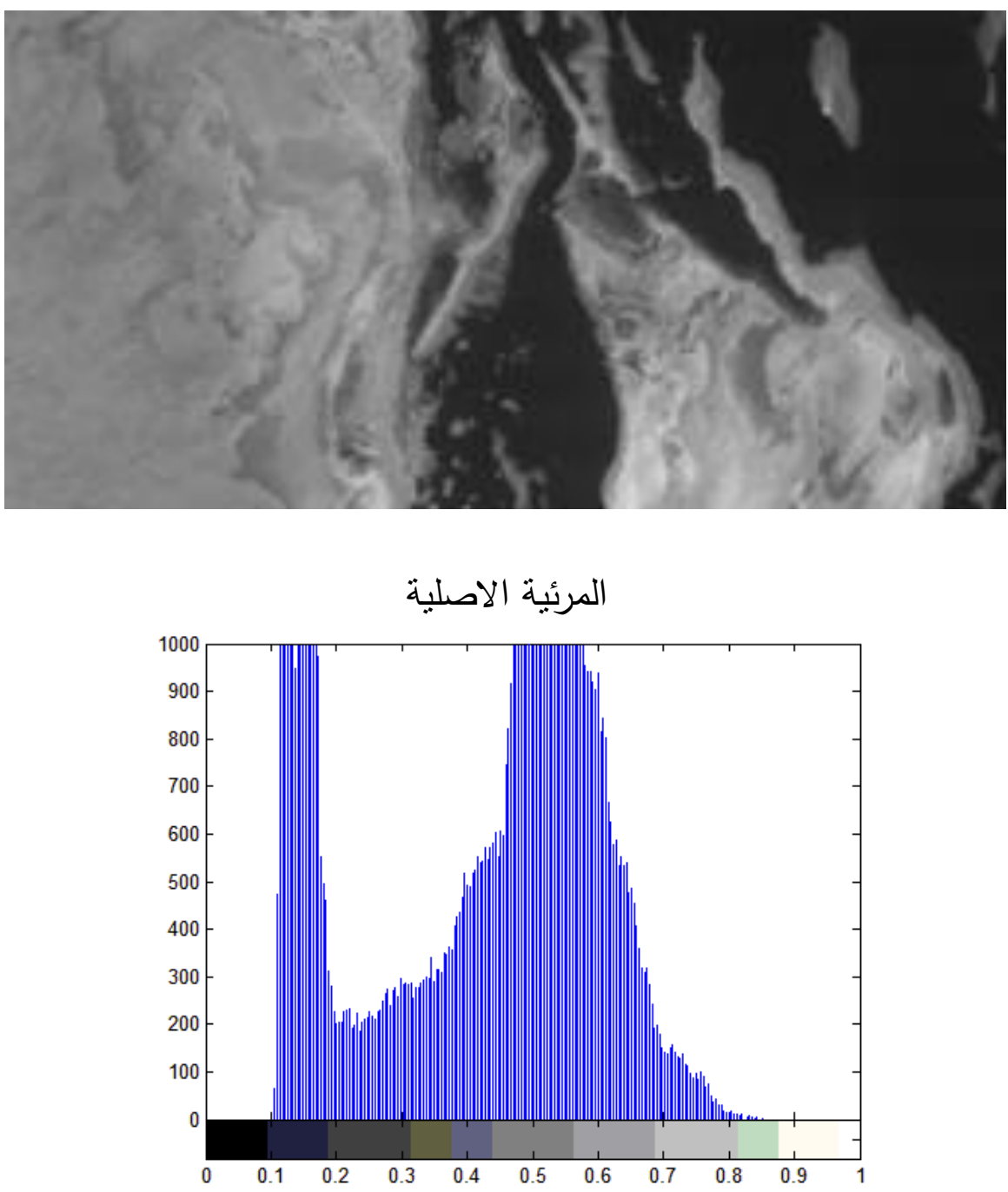

المدرج التكراري للمرئية قبل عملية الترشيح.

الثكل 2: يوضح المرئية المستخدمة والمدرج التكراري لها قبل المعالجة بمرشح الترددات الواطئة. 


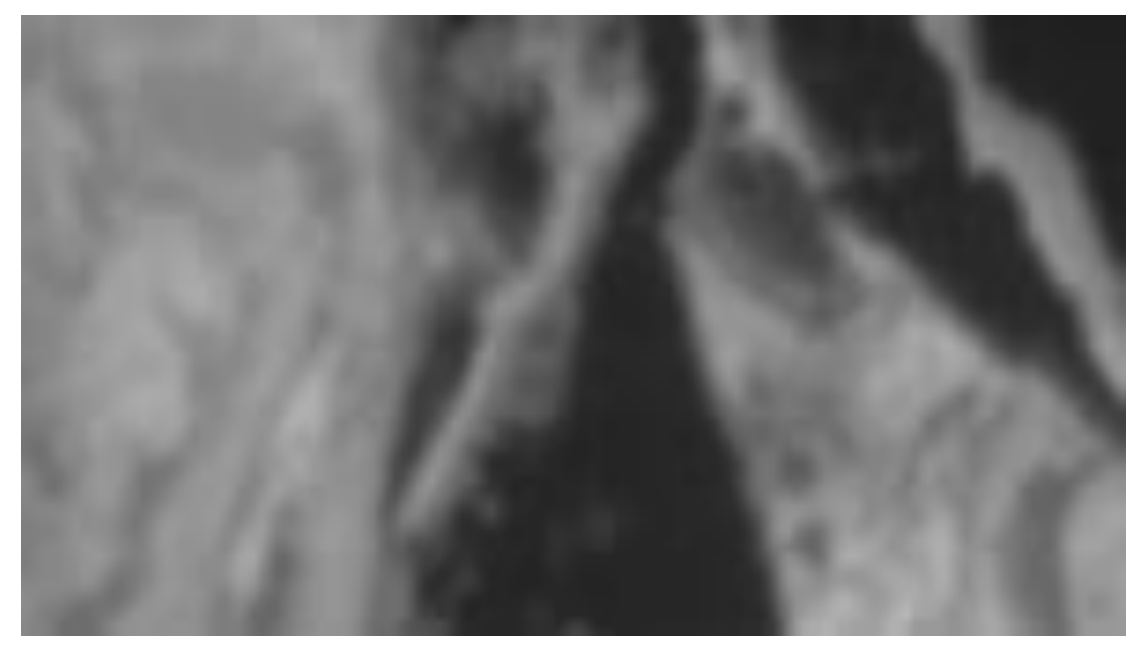

المرئية بعد معالجتها بمرشح الترددات الواطئة.

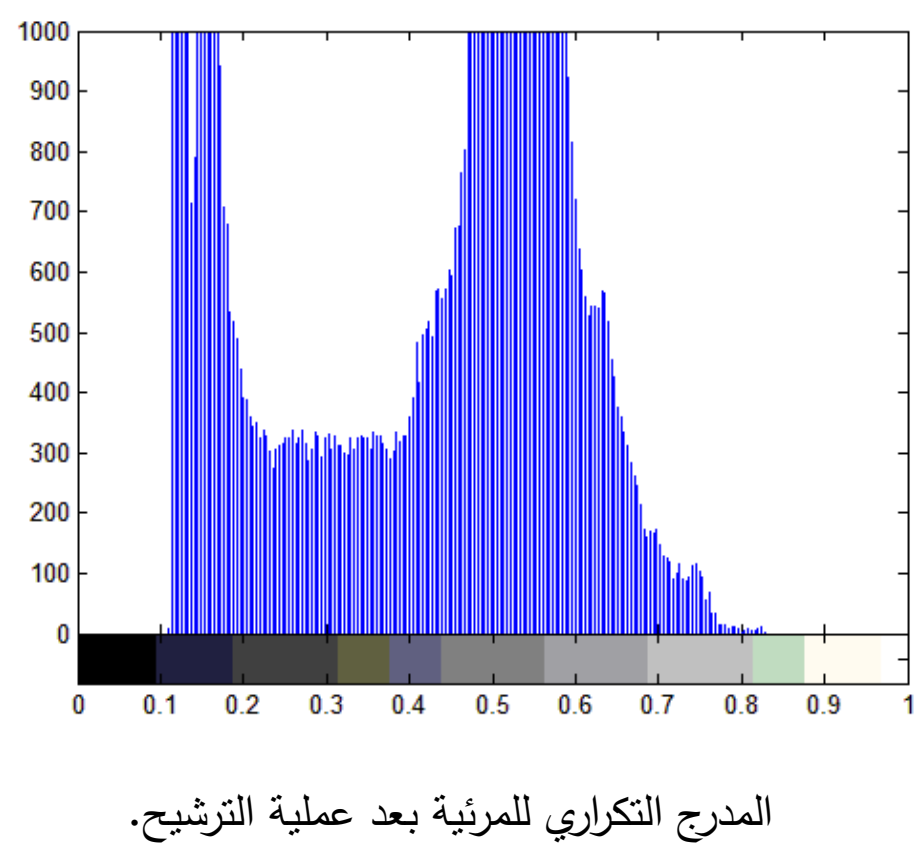

الثكل 3: يوضح المرئية المستخدمة والمدرج التكراري لها بعد المعالجة بمرشح الترددات الواطئة.

تم تقطيع المرئية باستخدام طريقة (Otsu) الى مرئيات تحنوي على صنفين، هما صنف المياه وصنف الاراضي اليابسة. حيث ان (الثكل 4) يوضح ناتج عملية تقطيع المرئية الاصلية و (الشكل 5) يوضح ناتج تقطيع المرئية الاصلية بعد معالجتها بمرشح كاوس لإمرار الترددات الواطئة. كما هو واضح من (الثكل 5) ان ناتج عملية تقطيع المرئية أكثر دقة بعد استخدام مرشح كاوس لإمرار الترددات الواطئة. حيث ان اللون الاسود يمنت المياهواللون الابيض يمنثل الاراضي اليابسة. 


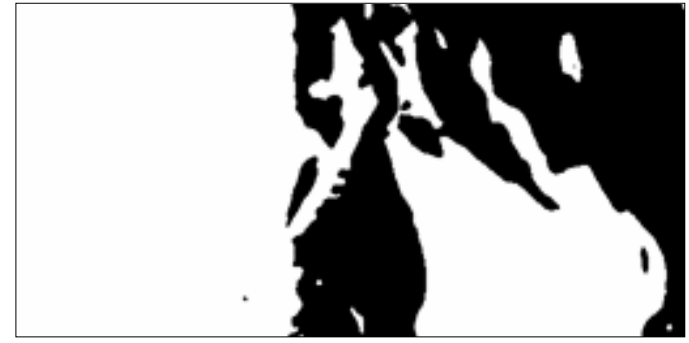

الثكل5: ناتج عملية تقطيع المرئية الاصلية بعد معالجنها بمرشح الترددات الواطئة.

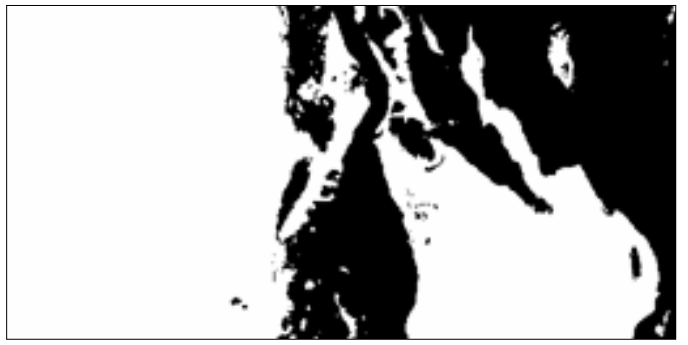

الثكل4: ناتج عملية تقطيع المرئية الاصلية.

بعد تقطيع المرئية الى صنفين تم تجزئة المرئية الى طبقتين (Layers). ونم خزن كل طبقة (صنف) في مرئية منفصلة ثنائية الالوان ابيض واسود (Binary image). اللون الابيض يمثل الصنف واللون الاسود ليس له قيمة. حيث الصنف الاول (Layer 1) يمثل المياه والصنف الثاني (Layer 2) يمنل الاراضي اليابسة، انظر (الثكل 6). لإنة.

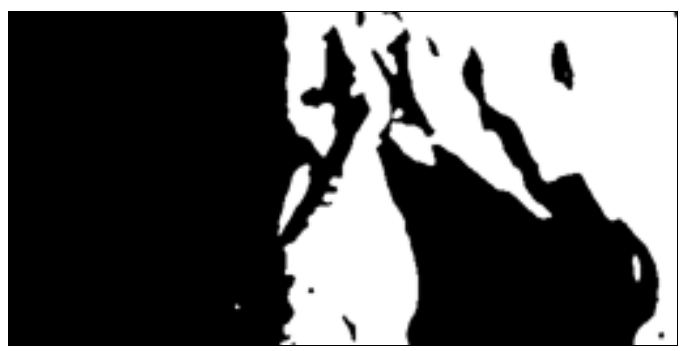

Layer 1

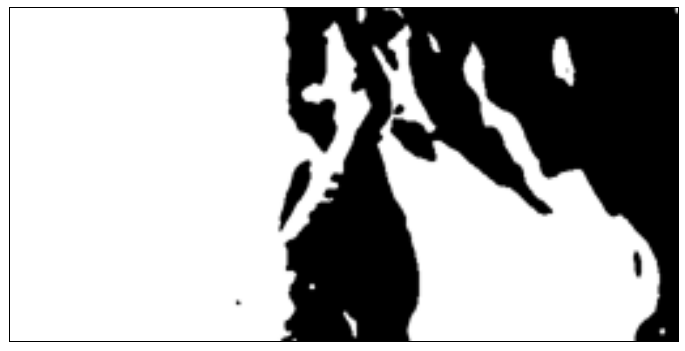

Layer 2

الثكل 6: تجزئة المرئية الى طبقتين (Layers).

تشير نتائج البحث الى انه نم تجزئة المرئية الاصلية التي تغطي منطقة البحر الاحمر الى منطقتين

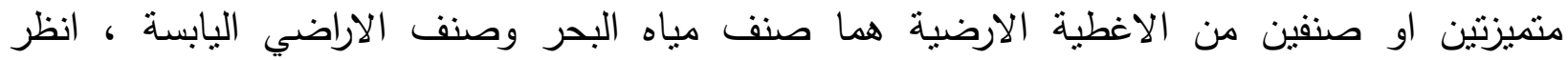

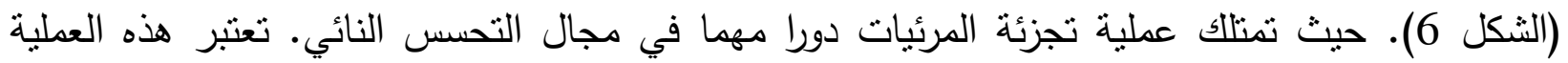

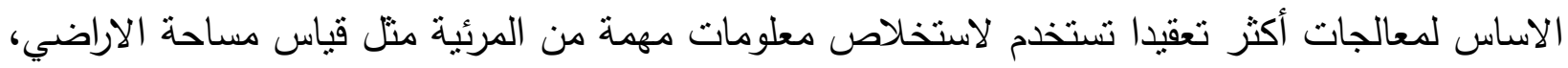




$$
\text { ضياء حازم سليمان البرهاوي }
$$

قياس طول المحيط لمنطقة معينة بالإضافة الى تحديد نوع الغطاء الارضي لكل منطقة متميزة. وهذه

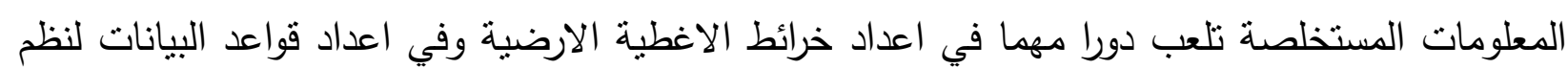

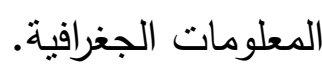

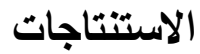

يمكن الخروج بالاستتاجات النالية من النتائج والملاحظات المذكورة اعلاه: 1- تمتاز طريقة حد العتبة (Otsu Thresholding) لتقطيع المرئيات (Image segmentation) بسرعة

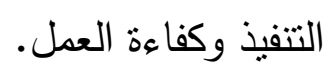

2- الاهية الكبيرة لعملية تقطيع المرئيات في تجزئة المرئية الى مناطق او اصناف متميزة يمكن اجراء

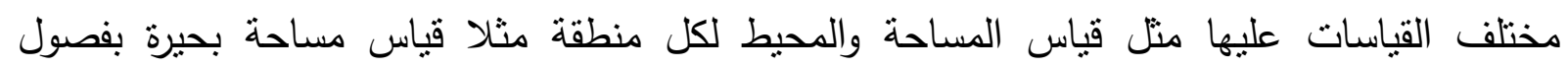
او سنوات مختلفة لإظهار مدى تزايد المياه او اليابسة على حد سواء.

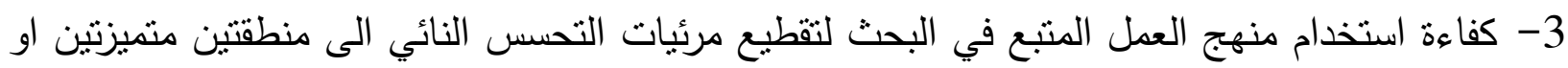
أكثر .

4- عملية نقطيع المرئيات لها اهمية كبيرة في اعداد خرائط تصنيف استعمالات الاراضي (Land use) وفي اعداد خرائط تصنيف الاغطية الارضية (Land cover) باستخدام مرئيات التحسس النائي.

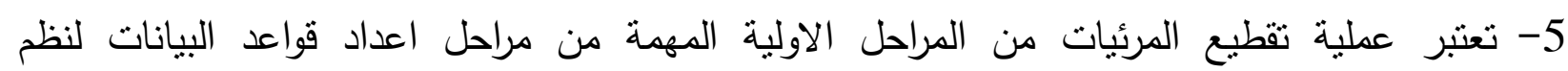

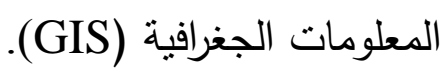

\section{المصادر الاجنبية}

Dey, V., Zhang Y., Zhong M., 2010. A Review On Image Segmentation Techniques with Remote Sensing Perspective, Iaprs Vol. xxxviii, Part 7A, Vienna, Austria, July 5 - 7, 2010.

Emre, Hamit Kok, Developing an Integrated System for Semi-Automated Segmentation of Remotely Sensed Imagery, Master thesis, Middle East Technical University, Turkey, 2005.

Gerhard X. Ritter and Joseph N. Wilson, Handbook of Computer Vision Algorithms in Image Algebra, Second Edition, CRC Press, 2001.

John, L. Semmlow, Biosignal and Biomedical Image Processing, published by Marcel Dekker, USA, 2004.

Nikhil, R. Pal and Sankar K. Pal, A Review on Image Segmentation Techniques, Pattern Recognition, , Pattern Recognition Society, Great Britain, Vol. 26, pp. 1277 - 1294, 1993.

Otsu, N., 1979, A Threshold Selection Method from Gray-Level Histograms, IEEE Transactions on Systems, Man and Cybernetics, Vol. 9, No. 1, 1979, pp. 62 - 66. 